\title{
Efeitos de amortecimento sobre um oscilador $\mathrm{X}^{3}$ \\ (Damping effects on $X^{3}$ oscillator)
}

\author{
A.J. Santiago ${ }^{1}$ e H. Rodrigues ${ }^{2}$ \\ ${ }^{1}$ Universidade do Estado do Rio de Janeiro, Rio de Janeiro, RJ, Brasil \\ ${ }^{2}$ Centro Federal de Educação Tecnológica, Rio de Janeiro, RJ, Brasil \\ Recebido em 20/10/2004; Revisado em 26/1/2005; Aceito em 2/2/2005
}

\begin{abstract}
Discutimos um método bastante simples de se introduzir o estudo do oscilador $x^{3}$ amortecido, tanto teórica como experimentalmente, em cursos elementares de Física. Dados experimentais do deslocamento de um oscilador $x^{3}$ em função do tempo, obtidos em medidas de ultrasom, são bem reproduzidos quando forças dissipativas são levadas em conta através de um único parâmetro.

Palavras-chave: oscilador $\mathrm{X}^{3}$, amortecimento, ensino de Física.
\end{abstract}

A simple method for introducing a damped $x^{3}$ oscillator in basic courses is discussed. The oscillator position as a function of time obtained from ultrason measurement are well reproduced in the case when the damping effects are taken into account through a single free parameter.

Keywords: $\mathrm{X}^{3}$ oscillator, dampping, Physics education.

\section{Introdução}

O estudo de movimentos anarmônicos é de grande interesse para Física. Em física da matéria condensada, o movimento vibracional de cada átomo em um cristal, em torno de um ponto de equilíbrio estável, pode ser descrito em termos de um movimento dentro do campo gerado por um potencial do tipo $U=c x^{2}-\alpha x^{3}-\beta x^{4}$ (não-harmônico) [1]. Já em física nuclear, o modo de respiração do movimento coletivo nuclear se mostra fortemente anarmônico, principalmente para núcleos leves [2]. Além disto, osciladores anarmônicos são excelentes laboratários para o estudo de caos determinístico $[3]$.

Apesar de toda esta aplicabilidade, movimentos anarmônicos dificilmente acham-se discutidos em livros textos usuais de Física, quer sejam eles de nível básico [4], quer sejam de nível intermediário [5] ou mesmo mais avançados $[6,7]$.

De fato, das referências citadas, apenas Landau e Lifchitz [7] dedicaram um capítulo para o estudo de oscilações anarmônicas. O livro deles, contudo, aborda o problema no contexto da formulação de Lagrange, formalismo este que não é apresentado em cursos de física básica [4]. Talves esta seja a principal motivação para diversos trabalhos que teem sido publicados sobre oscilações não-lineares [8-11].

Com efeito, esta falta de material didático a um nível mais elementar, sobre o problema das oscilações não-lineares, foi a principal motivação para este trabalho. Para ser mais específico, nosso objetivo neste trabalho é duplo. Primeiro, apresentar uma maneira bastante simples de se introduzir o oscilador $x^{3}$ amortecido em cursos elementares de física e segundo, reproduzir consistentemente os dados experimentais reportados por Cromer [8]. De fato, como veremos a seguir, Cromer construiu um dispositivo que permite ilustrar de uma maneira simples e bonita o oscilador $x^{3}$. Contudo, ao comparar seu modelo com os dados experimentais obtidos com medidas de ultrasom, um grande desvio entre teoria e experimento pode ser observado. Não obstante o valor ilustrativo do aparato de Cromer, é inegável que, do ponto de vista didático, é extremamente frustante para o aluno realizar o experimento e encontrar um desvio muito grande entre o modelo teorico e a medida experimental. Neste caso, é natural que desconfianças em relação à teoria e/ou ao experimento surjam no aluno. É verdade que poderiamos explorar apenas o carater qualitativo do experimento. Entretanto, mesmo assim, outras dificuldades inerentes à análise de experimentos qualitativos podem surgir [12]. Nesse sentido, experimentos que permitam ao aluno testar a teoria que lhe foi ensinada, podem melhor predispô-lo à compromissos epistemológicos tais como a coerência e a procura de generalizações .

\footnotetext{
${ }^{1}$ E-mail: ajsant@uerj.br. 
Este trabalho está organizado da seguinte maneira. Na Seção II, o problema do oscilador anarmônico é brevemente revisado, na Seção III, discutimos uma maneira bastante simples de se introduzir o problema do oscilador $x^{3}$ amortecido, tanto teórica como experimentalmente, em cursos mais elementares de Física. $\mathrm{Na}$ Seção IV, finalizamos com os resultados e as discussões.

\section{Oscilador anarmônico}

É bem conhecido que o movimento de uma partícula, para pequenos deslocamentos em torno de um ponto de equilíbrio estável, é harmônico simples. Por deslocamento pequeno, entendemos aquele para o qual termos de ordem maior ou igual a $x^{3}$, na expansão em série de Taylor da energia potencial em torno do ponto de equilíbrio, são negligenciáveis. Se esta condição não é satisfeita, então, o movimento é governado por uma equação do tipo

$$
m \ddot{x}=-k x-\alpha m x^{2}-\beta m x^{3}-\ldots
$$

onde $m$ é a massa e $k, \alpha$ e $\beta$ são coeficientes dos três primeiros termos da expansão, respectivamente. A seguinte notação será usada em todo este trabalho: $d x / d t=\dot{x}$ e $d^{2} x / d t^{2}=\ddot{x}$.

Neste caso, o movimento será ainda limitado e periódico mas não harmônico simples. Na verdade, movimentos deste tipo são chamados movimentos anarmônicos.

A Eq. (1) não é uma equação diferencial linear e, infelizmente, não existe uma método geral para resolver equações diferenciais não-lineares. Quando somente os termos não-lineares $x^{3}$ e $x^{4}$, na expansão em série da função energia potencial são retidos, soluções numéricas são facilmente obtidas para o problema uma vez que integrais do tipo $\int_{x} d x\left[E-k x^{2} / 2-m \alpha x^{3} / 3-m \beta x^{4} / 4\right]^{1 / 2}$ são expressas em termos de integrais elipticas que têm seus valores tabelados. Por outro lado, se os termos $\alpha x^{3}$ e $\beta x^{4}$ são pequenos, soluções aproximadas podem ser obtidas por vários métodos. Um método comumente empregado consiste em considerar os termos não-lineares como termos de não-homogeneidade em equações do tipo

$$
\ddot{x}+w_{0}^{2} x=-\beta x^{3},
$$

onde, por simplicidade admitimos $\alpha=0$.

Assim, a solução não-homogênea da Eq. (2) pode ser expressa em termos da função de Green [13]

$$
G(t, \zeta)= \begin{cases}\sin w_{0}(t-\zeta), & t \geq \zeta \\ 0, & t \leq \zeta\end{cases}
$$

e, então, a solução particular da Eq. (2) é (note que a Eq. (2) é uma equação não-linear e então, sua solução geral não pode ser escrita como a soma da solução para $\beta=0$ mais a solução para $\beta \neq 0)$ :

$$
x(t)=-\int d \zeta \beta x^{3}(\zeta) \frac{\sin w_{0}(t-\zeta)}{w_{0}} .
$$

Como o movimento que estamos considerando é periódico, a solução pode ser expressa em termos da expansão de Fourier para $x(\zeta)$, e a primeira aproximação para a solução é

$$
x_{1}(\zeta)=a_{1} \cos w \zeta
$$

que, levada a equação integral (Eq. (4)) fornece

$$
x(t)=a_{1} \cos w t+a_{3} \cos 3 w t,
$$

$\operatorname{com} w=\sqrt{w_{0}^{2}+3 \beta a_{1}^{2} / 4}$ e $a_{3}=\beta a_{1}^{3} / 32 w_{0}^{2}$.

O lado direito da Eq. (6) pode ser considerado nova aproximação para $x(\zeta)$, levado à Eq. (4) e, desta forma, nova solução é obtida com melhor aproximação, e assim sucessivamente. Métodos deste tipo, para obter soluções de equações diferenciais, são conhecidos como métodos de aproximações sucessivas.

\section{Construindo um oscilador $\mathrm{X}^{3}$}

\subsection{O dispositivo de Cromer}

Podemos construir um oscilador $x^{3}$ prendendo um planador sobre um trilho de ar à uma mola como ilustra a Fig. 1(a). A distância $L$ entre o suporte da mola e o planador deve ser exatamente igual ao comprimento $L_{0}$ da mola quando sujeita apenas a tração de seu próprio peso. Isto é, o trilho de ar deve ser ajustado de maneira a compensar o peso do planador. Quando o planador é deslocado de sua posição de equilíbrio de uma distância $x$ (Fig. 1(b)), o comprimento da mola aumentará de $\sqrt{L_{0}^{2}+x^{2}}-L_{0}$, tal que a força restauradora $F$ da mola será

$$
F=-k L_{0}\left(\sqrt{1+\left(\frac{x}{L_{0}}\right)^{2}}-1\right) .
$$

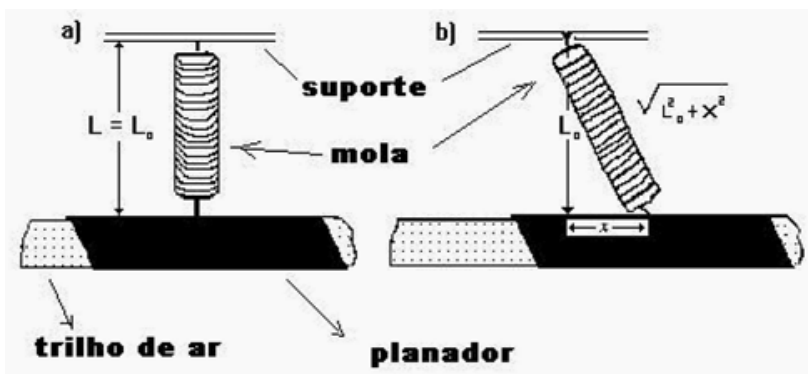

Figura 1 - O Dispositivo de Cromer com o planador na posição de equilibrio (a) e deslocado horizontalmente (b).

Para pequenos valores do deslocamento $x$, em que $x<<L_{0}$, vemos que

$$
\sqrt{1+\left(\frac{x}{L_{0}}\right)^{2}} \approx 1+\frac{1}{2}\left(\frac{x}{L_{0}}\right)^{2}
$$


$\log 0$

$$
F=-\frac{k x^{2}}{2 L_{0}}
$$

A componente da força da mola na direção $x$ será $F_{x}=F \cos \theta \operatorname{com} \cos \theta=x / \sqrt{L_{0}^{2}+x^{2}} \approx x / L_{0}$ e, portanto,

$$
F_{x}=-\kappa x^{3},
$$

$\operatorname{com} \kappa=k / 2 L_{0}^{2}$

A Eq. (10) nos diz que dentro da aproximação considerada o planador é um oscilador $x^{3}$. Aproximações como as que adotamos acima são comumentes usadas para o movimento pendular, i.e., na mesma aproximação que um movimento pendular é harmônico simples o planador é um oscilador $x^{3}$.

\subsection{O efeito do amortecimento}

Dispositivos como o descrito na seção anterior têm sido sugerido por Cromer [8] no estudo do período do oscilador $x^{3}$. Em particular, na Ref. 8, dados experimentais do deslocamento em função do tempo de um oscilador $x^{3}$, que foram obtidos através de medidas com um sistema de ultrasom [14], foram reportados. Estes resultados foram compilados da Ref. [8] e encontram-se agrupados na Tabela 1.

Tabela 1 - $x$ como função de $t$ do oscilador $x^{3}$. Dados compilados da Ref. [8].

\begin{tabular}{crrr}
\hline$t(s)$ & $x(m)$ & $t(s)$ & $x(m)$ \\
\hline 0.1 & 0.058 & 0.2 & 0.047 \\
0.3 & 0.030 & 0.4 & 0.005 \\
0.5 & -0.011 & 0.6 & -0.032 \\
0.7 & -0.048 & 0.8 & -0.058 \\
0.9 & -0.057 & 1.0 & -0.047 \\
1.1 & -0.030 & 1.2 & -0.010 \\
1.3 & 0.009 & 1.4 & 0.028 \\
1.5 & 0.042 & 1.6 & 0.048 \\
1.7 & 0.045 & 1.8 & 0.036 \\
1.9 & 0.023 & 2.0 & 0.008 \\
2.1 & -0.007 & 2.2 & -0.023 \\
2.3 & -0.039 & 2.4 & -0.052 \\
2.5 & -0.055 & 2.6 & -0.051 \\
2.7 & -0.041 & 2.8 & -0.028 \\
2.9 & -0.012 & 3.0 & 0.002 \\
3.1 & 0.016 & 3.2 & 0.027 \\
3.3 & 0.032 & 3.4 & 0.036 \\
\hline
\end{tabular}

O modelo teórico de Cromer para o oscilador $x^{3}$, todavia, não pode fornecer uma boa descrição dos dados experimentais que, nitidamente, diminuíam de amplitude em função do tempo. Na realidade, todo movimento macroscópico é amortecido e uma comparação realística com dados experimentais deve, necessariamente, levar em conta este efeito. Não é difícil imaginar que o amortecimento é produzido pelo atrito do meio em que o corpo se desloca. O atrito sendo uma força dissipativa fará com que a energia do corpo em movimento transforme-se em calor, i.e., dissipa-se. Nestas condições, o processo de movimento já não é puramente mecânico, e uma análise rigorosa requer que se leve em conta o movimento do próprio meio e do estado térmico interno do sistema (corpo + meio). Existem alguns casos, entretanto, em que o movimento num meio dissipativo pode ser descrito, aproximadamente, através das equações mecânicas de movimento. Nestes casos, em geral, podemos considerar que sobre o corpo atua uma força de atrito dependente de sua velocidade. Se, além disto, a velocidade é suficientemente pequena, podemos desenvolver a força de atrito em uma série de potências. Como o termo de ordem zero do desenvolvimento será nulo (sobre um corpo parado não atua força de atrito), o primeiro termo não nulo será proporcional e de sentido contrário à velocidade, i.e., chamando de $b$ o coeficiente de proporcionalidade temos,

$$
F_{a}=-b \dot{x} .
$$

Assim, a equação de movimento para o planador, levando-se em conta o amortecimento, é

$$
F_{x}=-\kappa x^{3}-b \dot{x} .
$$

Nas Eqs. (11) e (12), b depende de vários fatores tais como o meio, a forma do corpo e etc. Neste trabalho, consideraremos $b$ um parâmetro que será ajustado aos dados experimentais. Naturalmente, $\operatorname{com} b=0$, a Eq. (12) se reduz à Eq. (10).

\subsection{Integração das equações de movimento}

A equação de movimento do oscilador $x^{3}$ amortecido (Eq. (12)) pode ser reescrita como

$$
\ddot{x}=-\frac{\kappa}{m} x^{3}-\frac{b}{m} \dot{x},
$$

e, então, separada em duas equações diferenciais de primeira ordem. As equações assim obtidas podem ser facilmente integradas em um intervalo de tempo $\Delta t$. Assim, obtemos

$$
x(t+\Delta t)=x(t)+v(t) \Delta t
$$

e

$$
v(t+\Delta t)=v(t)-\left[\frac{\kappa}{m} x^{3}(t)+\frac{b}{m} v(t)\right] \Delta t
$$

Este método de integração, por sua simplicidade e por fornecer resultados com boas aproximações tem sido fartamente usado na literatura [15]. De fato, Eqs. (14) e (15) fornecem bons resultados para o problema desde que $\Delta t$ seja escolhido suficientemente pequeno quando comparado com o período do oscilador $x^{3}$ em questão.

Obviamente, o método de aproximações sucessivas (Seção II) poderia ser empregado neste caso. Todavia, em geral, nos cursos introdutórios de Física, embora o aluno muito provavelmente já tenha visto cálculo numérico e, então, tenha noções de Fortran, Pascal, Basic, etc..., ainda não está familiarizado com métodos 
da física-matemática. Assim, apresentar um método numérico nos parece mais interessante. Nada impede, todavia, que em cursos mais avançados, o método algébrico apresentado na seção anterior seja também realizado e comparado com o método numérico.

\section{Resultados e discussões}

Para evoluir dinâmicamente o sistema, as Eqs. (14) e (15) são resolvidas iterativamente com $\Delta t=0,01 \mathrm{~s}$ durante $5 \mathrm{~s}$. Nós checamos que os resultados ficam praticamente inalterados se $\Delta t \leq 0,05 \mathrm{~s}$. Os resultados foram obtidos para um planador com $L_{0}=0,12 \mathrm{~m}$, $k=70 \mathrm{~N} / \mathrm{m}$ e $m=0,439 \mathrm{~kg}$.

A Fig. 2 traz os resultados para a posição $x$ (Fig. 2(a)) e a velocidade $v$ (Fig. 2(b)) quando plotados em função de $t$. Três casos são considerados, oscilador $x^{3}$ com amplitude $A=0,06 \mathrm{~m}$ (linha sólida), oscilador $x^{3}$ com amplitude $A=0,03 \mathrm{~m}$ (linha pontilhada) e oscilador linear $(F=-k x)$ com amplitude $A=0,06 \mathrm{~m}$ (linha tracejada). Nota-se que, há uma grande diferença de fase e de período entre o oscilador $x^{3}$ e o linear. Além disto, pode-se observar que o período $\tau$ do oscilador $x^{3}$ depende da amplitude de oscilação e, na verdade, o período do oscilador $x^{3}$ é inversamente proporcional à amplitude de oscilação.
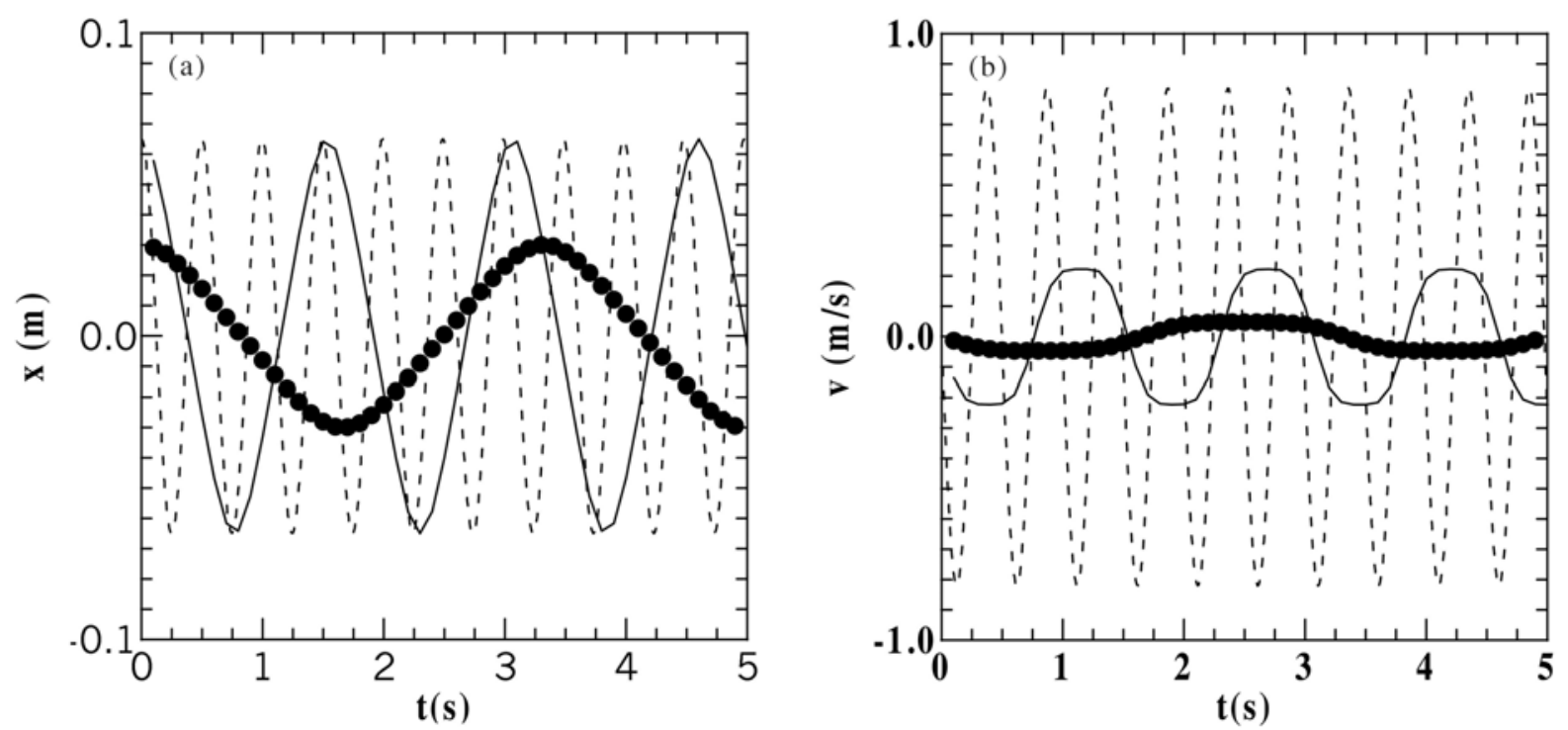

Figura 2 - A posição $x$ (a) e a velocidade $v(\mathrm{~b})$ do planador $v s$. $t$. A linha sólida representa os resultados para o oscilador $x^{3}$ com amplitude $A=0,06 \mathrm{~m}$, a pontilhada com $A=0,03 \mathrm{~m}$ e a tracejada os resultados para o oscilador linear com $A=0,06 \mathrm{~m}$.

Na Fig. 3, são apresentados $x$ (Fig. 3(a)) e $v$ (Fig. $3(\mathrm{~b})$ ) do oscilador $x^{3}$ como uma função do tempo, para vários valores do parâmetro $b$. A linha sólida corresponde a $b=0$, as cruzes $b=0,01$, os triângulos cheios $b=0,1$, os triângulos vazios $b=0,5 \mathrm{e}$, os quadrados vazios $b=1$. Por simplicidade, na Fig. 3(b) somente os resultados para $b=0$ e $b=0,1$ são apresentados. Observa-se que o amortecimento provoca diferentes mudanças nas curvas. Primeiro altera o valor da amplitude de oscilação em função do tempo e, segundo, modifica o período de oscilação. Em particular, quanto maior o $b$, maior é o período de oscilação e menor a amplitude de oscilação em função do tempo (resultado similar é observado em $v \times t$ ). Este resultado pode ser facilmente entendido uma vez que o amortecimento é uma força dissipativa e, portanto, consome a energia do sistema a qual é proporcional a amplitude. Um decréscimo na amplitude provoca aumento no período pois, como já mencionamos, o período de oscilação é inversavemente proporcional à amplitude.

Finalmente, na Fig. 4, nosso melhor ajuste (linha sólida) para os dados experimentais (triângulos) da Ref. 8 (cf. Tabela 1) são apresentados. Por completeza, os resultados de Cromer (linha tracejada), sem levar em conta o amortecimento $(b=0)$, são também mostrados. Podemos observar que, enquanto os resultados para $b=0$ se desviam cada vez mais dos resultados experimentais para $t$ crescente, a inclusão do efeito de amortecimento permite uma boa reprodução dos dados experimentais. A pequena diferença residual entre os valores téoricos e experimentais pode ser diminuída ainda mais se termos de ordem superior forem mantidos nas expansões utilizadas. 

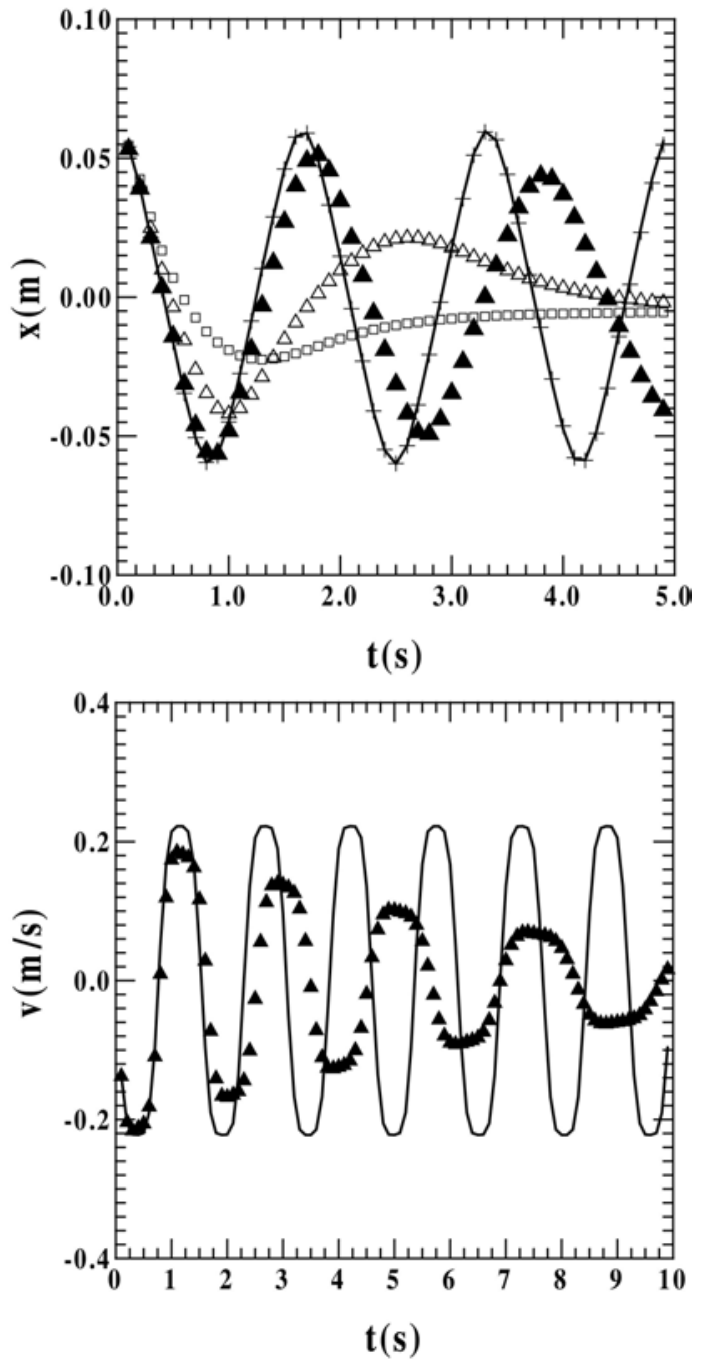

Figura $3-x$ (a) e $v$ (b) vs. $t$ para $b=0$ (linha sólida), $b=0,01$ (cruzes), $b=0,1$ (triângulos cheios), $b=0,5$ (triângulos vazios) e, $b=1$ (quadrados vazios). Por simplicidade, na Fig. 3(b) somente os resultados para $b=0$ e $b=0,1$ são apresentados.

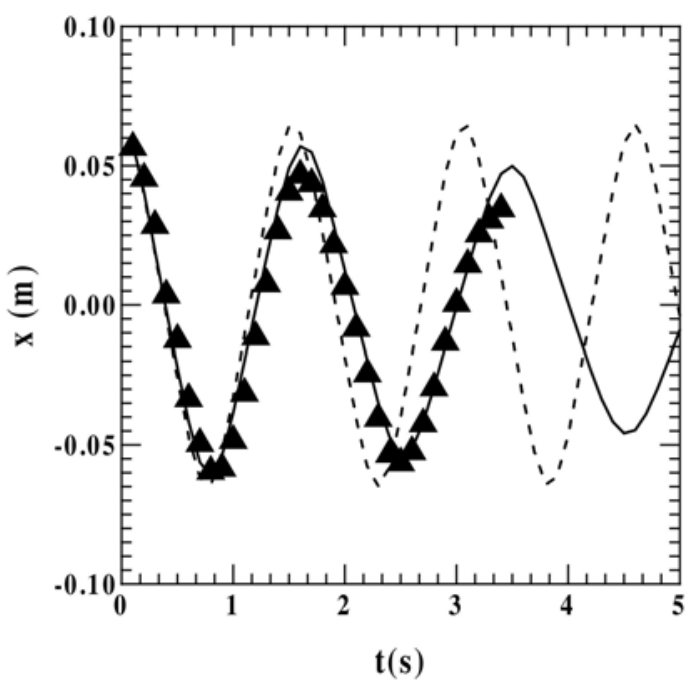

Figura 4 - $x$ vs. $t$ para o oscilador $x^{3}$. A linha sólida representa nossos resultados, os triângulos os dados experimentais da Ref. 8 , e a linha tracejada os resultados de Cromer [8].
Em suma, discutimos um método bastante simples para estudar o oscilador $x^{3}$ amortecido. A discussão apresentada é simples o bastante para permitir a inclusão do estudo de osciladores anarmônicos tanto teórica quanto experimentalmente, em cursos técnicos (Física, Engenharia, Matemática...) mais elementares, onde por exemplo, livros textos no nível de Halliday e Resnick [4] forem adotados. Especial ênfase foi dada ao efeito de forças dissipativas em medidas experimentais. Em particular, mostramos que para um certo valor do parâmetro de amortecimento os dados experimentais ficam muito bem reproduzidos.

Finalmente, gostaríamos de chamar a atenção do leitor para o fato de que nossa abordagem simplificou drásticamente o problema das oscilações não-lineares. Em partícular somente o termo em $x^{3}$ foi considerado, e um estudo mais rigoroso devería considerar termos em $x^{2}$ e termos de ordens mais altas acoplados ao termo linear. Contudo, o tratamento apresentado acima pode ser didaticamente útil na apresentação do problema das oscilações não-lineares em cursos básicos de Física.

\section{Referências}

[1] C. Kittel, Introdução à Física do Estado Sólido (Ed. Guanabara Dois, Rio de Janeiro, 1978), $5^{\text {a }}$ edição, cap. 5.

[2] J.P. Blazot, J.F. Berger, J. Dechargé e M.Girod, Nucl. Phys. A591, 435 (1995). C.S. Wang, K.C. Chung e A.J. Santiago, Phys. Rev. C55, 2844 (1997).

[3] M.A.M. de Aguiar, Rev. Bras. Ens. Fis. 16, 3 (1994). P.W. Laws, Am. J. Phys. 72, 446 (2004).

[4] H. Moysés Nussenzveig, Curso de Física Básica (Ed. Edgard Blücher Ltda., Sâo Paulo, 1981), v. 2. D. Halliday e R. Resnick, Fundamentos de Física (Ed. Livros Técnicos e Científicos Editora S.A., Rio de Janeiro, 1994), v. 2, $3^{\text {a }}$ edição.

[5] K.R. Symon, Mecânica, (Ed. Campus Ltda., São Paulo, 1982), $5^{\mathrm{a}}$ edição.

[6] H. Goldstein, Classical Mechanics (Ed. AddisonWesley Publishing Company, Massachusetts, 1981), $2^{\mathrm{a}}$ ẽdição.

[7] L. Landau e E. Lifchitz, Mécanique (Ed. Éditions de la Paix, Paris, 1978), v. I.

[8] A. Cromer, The Phys. Teacher 30, 249 (1992).

[9] I.R. Gatland, Am. J. Phys. 59, 155 (1991).

[10] B. Pecori, G. Torzo e A. Sconza, Am. J. Phys. 67, 228 (1999).

[11] P.B. Kahn e Y. Zarmi, Am. J. Phys. 72, 538 (2004).

[12] A. Villani e L. Orquiza de Carvalho, Rev. Bras. Ens. Fis. 16, 98 (1994).

[13] E. Butkov, Física Matemática (Ed. Guanabara Dois S.A., Rio de Janeiro, 1983).

[14] R. Gatland, Computers in Physics 5, 541 (1991).

[15] J.P. Bondorf, R. Donangelo and K. Sneppen, Phys. Lett. B214, 321 (1988). 